\title{
Surgical pathology associated with Meckel's diverticulum in a tertiary hospital. 12 year review
}

\author{
Cristina Méndez García ${ }^{1}$ J. M. Suárez Grau², C. Rubio Chaves ${ }^{1}$, J. A. Martín Cartes ${ }^{1}$, \\ Fernando Docobo Durántez ${ }^{1}$ and F. J. Padillo Ruiz ${ }^{1}$ \\ Unit of Clinical Management of General and Digestive Surgery. ${ }^{1}$ Hospital Universitario Virgen del Rocío. Seville, Spain. \\ ${ }^{2}$ Hospital General Básico de Riotinto. Huelva, Spain
}

\begin{abstract}
Objective: we want to present our experience about surgical pathology of Meckel's diverticulum by means of a retrospective study.

Material and methods: we report a group of patients of our Department of General and Abdominal Surgery and Paediatric Surgery of our Hospital with Meckel's diverticulum since January of 1997 to January of 2010 . We report the clinical presentation, complementary test, interventions, and the postoperative follow up.

Results: 45 patients were operated in total, 33 of them in emergency surgery under the clinical form of acute abdominal pain; and the others 12 in programmed surgery, these cases came up more frequently like a clinical manifestations of latent abdominal pain, rectal bleeding and anaemia. The complementary tests were so varied; abdominal ultrasounds were used in $63 \%$ of emergency cases and the $40 \%$ of programmed cases, in these patients, gammagraphy with Tc99 was the second test in frequency. Laparoscopy was used in 10 cases $(22 \%)$. The main surgery technique used was diverticulectomy (82\%).

Conclusions: the presence of Meckel's diverticulum has to be clinically suspected in all patients with abdominal pain of unknown aetiology. Access to the abdominal cavity using routine laparoscopy provides essential information on the diagnosis and for the treatment.
\end{abstract}

Key words: Diverticulum. Meckel. Surgery. Laparoscopy.

Méndez García Cristina, Suárez Grau J. M, Rubio Chaves C, Martín Cartes J. A, Docobo Durántez Fernando, Padillo Ruiz F. $J$. Surgical pathology associated with Meckel's diverticulum in a tertiary hospital. 12 year review. Rev Esp Enferm Dig 2011; 103: 250-254.

Received: $18-10-10$

Accepted: 04-02-11.

Correspondence: Cristina Méndez García. General and Digestive Surgery Unit. Hospitales Universitarios Virgen del Rocío. Avda. Manuel Siurot, s/n. 41013 Sevilla. Spain

e-mail: cmendicea@yahoo.es

\section{INTRODUCTION}

Meckel's diverticulum (MD) is a vestigial remnant of the omphalomesenteric duct of the foetal digestive tract, which was not fully reabsorbed before birth. Its prevalence is approximately $2 \%$ in the general population, and in most cases is asymptomatic. However, these diverticula can become infected (diverticulitis) in a small number of patients, causing intestinal blockage or bleeding of the mucosa. Meckel's diverticulitis is frequently misdiagnosed as appendicitis. It usually presents in the first years of life, but can also occur in adults.

The objective of this study was to show the prevalence of this disease in our area and perform a systematic review of the literature.

\section{MATERIAL AND METHODS}

We present a series of 45 cases undergoing surgery in the general and paediatric surgery units of our hospital between January 1997 and January 2010. We reviewed all patient records and analysed each patient's features, presentation of the disease, diagnostic resources used, treatment received, complications and follow-up to final healing.

\section{RESULTS}

A total of 45 patients aged between 0 and 68 years were operated on. Thirty-three of them underwent emergency surgery and 12 scheduled surgery; and in 10 cases (22\%), laparoscopy was used (4 emergency surgery cases and 6 scheduled surgery cases).

Many of the patients had no history of the condition and where it did exist, it was very varied, and no causal relationship could therefore be established. 


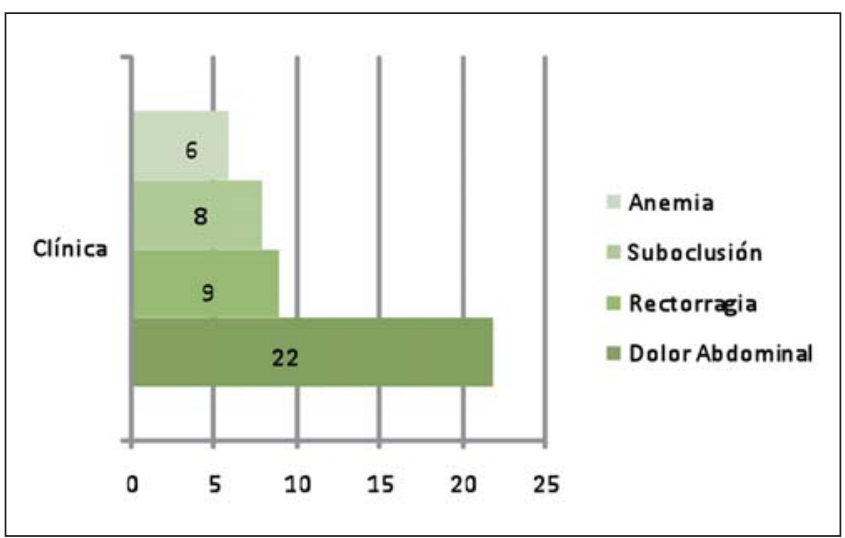

Fig. 1. Clinical manifestations.

The clinical symptoms of the disease (Fig. 1) included non-specific abdominal pain with malaise, vomiting and fever, acute abdominal pain and lower gastrointestinal bleeding, sometimes with hemodynamic compromise.

In 26 cases there was no previous suspicion of diverticular disease, while in 19 cases Meckel's diverticulum was suspected due to initial symptoms. The diverticulum was the cause of the disease and required surgery in all these cases.

All patients underwent basic laboratory tests (abdominal and thorax X-rays, biochemical analysis, blood count and coagulation study) as part of the preoperative evaluation.

The diagnostic tests were varied. Emergency surgery was indicated by the clinical symptoms in $37 \%$ of patients, while in others, abdominal ultrasound was performed. Only in 2 selected cases computed tomography (CT) was used. In less acute cases, where the disease was suspected and it was possible to complete the study for a specific diagnosis, endoscopy, colonoscopy, scintigraphy with $\mathrm{Tc}^{99}$ and arteriography were subsequently used (Fig. 2).

A detailed description is as follows:

1. Abdominal ultrasound performed in 16 patients: 7 adults and 9 children (a clinical diagnosis of Meckel's diverticulum was established in $50 \%$ of these cases).

2. Barium studies: A barium gastrointestinal transit test in one adult with suspected Meckel's diverticulum showed a negative result.

3. Abdominal CT: 5 in total, 4 in children without diagnosing the diverticular disease, and 1 in an adult patient, which established the diagnosis of MD.

4. Magnetic resonance imaging: In only 1 child, without obtaining a diagnosis of MD.

5. Oral endoscopy: 7 oral endoscopies, 2 in adults with suspected diverticular disease (for detecting higher rectal bleeding) and 5 in children under 18 .

6. Scintigraphy with $\mathrm{Tc}^{99}$ : In 6 patients, 1 adult and 5 children due to clear suspicion of MD (the test was

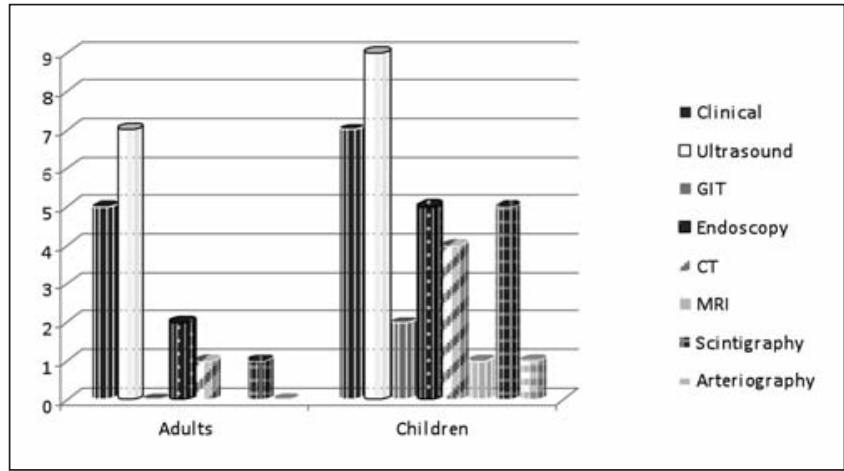

Fig. 2. List of diagnostic tests requested.

negative in $50 \%$ of cases, but these cases later proved positive for the disease, indicating the high rate of false negatives in this test).

7. Arteriography: In 1 child with suspected MD (the same case as in the scintigraphy, which despite the negative test results, An scheduled intervention was decided, as an ulcerated Meckel's diverticulum was the cause of the abdominal pain and weight loss).

The surgical technique performed was diverticulectomy and enterorrhaphy in 37 patients $(82.2 \%)$ (Figs. 3, 4 and 5); while in 8 cases (17.8\%) resection of the ileal segment and termino-terminal anastomosis was required.

Prophylactic appendectomy was associated with the disease in 23 patients (slightly more than $50 \%$ ), and 10 of them were children. The lesion in all cases was confined to the ileal segment of the small intestine and Meckel's diverticulum was confirmed after the relevant anatomopathological study.

All patients underwent surgery successfully, with remission of the symptoms being achieved in all cases. Postoperative complications were as follows:

- 2 obstructions due to adhesions requiring re-operation.

- 1 evisceration requiring intervention.

- 1 case of intolerance to suture of the abdominal wall.

This represents a complication rate of less than $1 \%$.

The follow-up time was 3-4 months for children (aged less than 18 years) and longer (4-12 months) for adults. There was no recurrence of the disease.

\section{DISCUSSION}

Meckel's diverticulum is the most common congenital anomaly of the gastrointestinal tract. We found an incidence of $2-3 \%$ in the general population (1) after reviewing a series of patients undergoing surgery, and $0.3 \%$ for research findings at autopsy (2). MD stems from the incomplete atrophy of the omphalomesenteric duct, which normally closes from the tenth week of embryological development and becomes a fibrous band that disappears (2). As well as MD, other secondary abnormalities due to 


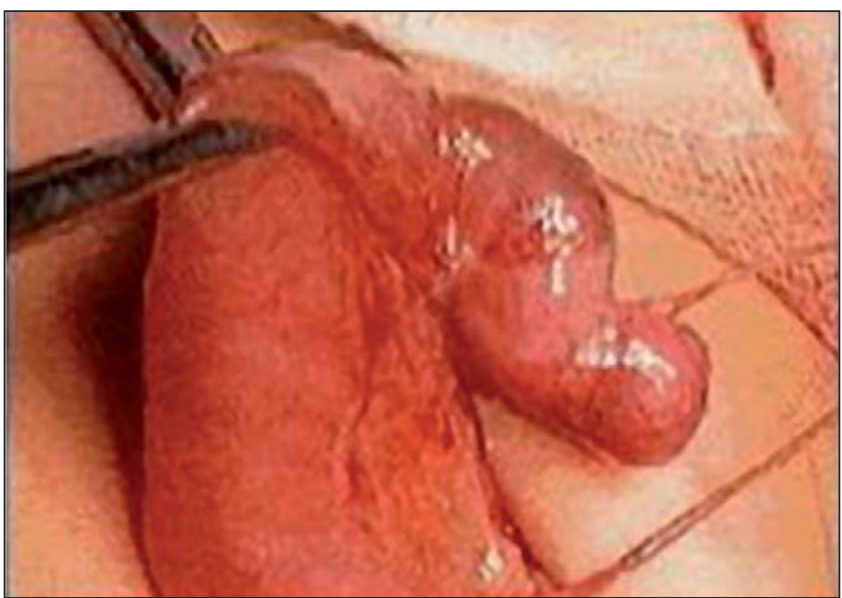

Fig. 3. Symptomatic Meckel's diverticulum in the distal ileal region.

the incomplete regression of the omphalomesenteric duct are umbilico-ileal fistula, umbilical sinus and the presence of a hard fibrous cord. DM is by far the most frequent of these conditions (up to 98\%) (2).

The most common site for DM is the antimesenteric border of the terminal ileum, $60-100 \mathrm{~cm}$ from the ileocecal valve in adults, and $30-90 \mathrm{~cm}$ in children.

Large series for DM, both at autopsy and during surgical procedures, show no differences between the sexes (3). However, for symptomatic DM, the incidence is somewhat higher in men than in women (1.6-1.8:1) (4). Although no association between DM and other major congenital malformations has been demonstrated, a higher incidence of patients with Crohn's disease (CD) has been found (up to 5-8\% of patients with $\mathrm{CD}$, depending on the series) (5).

Anatomopathologically, DM is considered as a diverticulum, as it includes all layers of the intestinal wall. Up to $50 \%$ of DM cases have a different tissue formation from the adjacent ileum, with the most frequent being

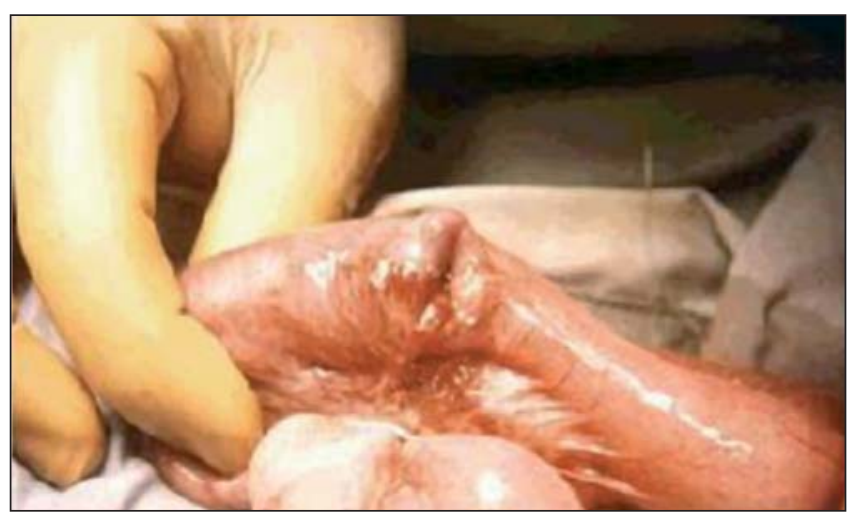

Fig. 4. Enterorrhaphy after simple diverticulectomy.

gastric mucosa (23-50\%) (6). It also may present pancreatic tissue $(5-16 \%)$ (7) or exceptionally biliary or colonic epithelium (2).

There are studies of tumours lodging in the DM lumen, which may be benign, such as leiomyomas, angiomas, or neurofibromas, or malignant, such as leiomyosarcomas or carcinoid tumours (2).

Most DM cases are asymptomatic, and may be an incidental finding during imaging studies or surgery for other reason.

Lower gastrointestinal bleeding is the most common clinical manifestation, especially in children under 2 years (8) (ranging from positive faecal occult blood to massive obvious bleeding). Therefore up to $50 \%$ of cases show ulceration of the gastric mucosa housed inside (2). The second most common manifestation is intestinal obstruction, mainly in adolescents and adults $(2,9)$. This appears as a mechanical obstruction of the small intestine with distension, diffuse abdominal pain, vomiting and constipation, the pathophysiological mechanism being a volvulus or intussusception (2). Incarceration of a DM in a hernia sac is known as Littre's hernia. Up to 50\% of these cases occur in

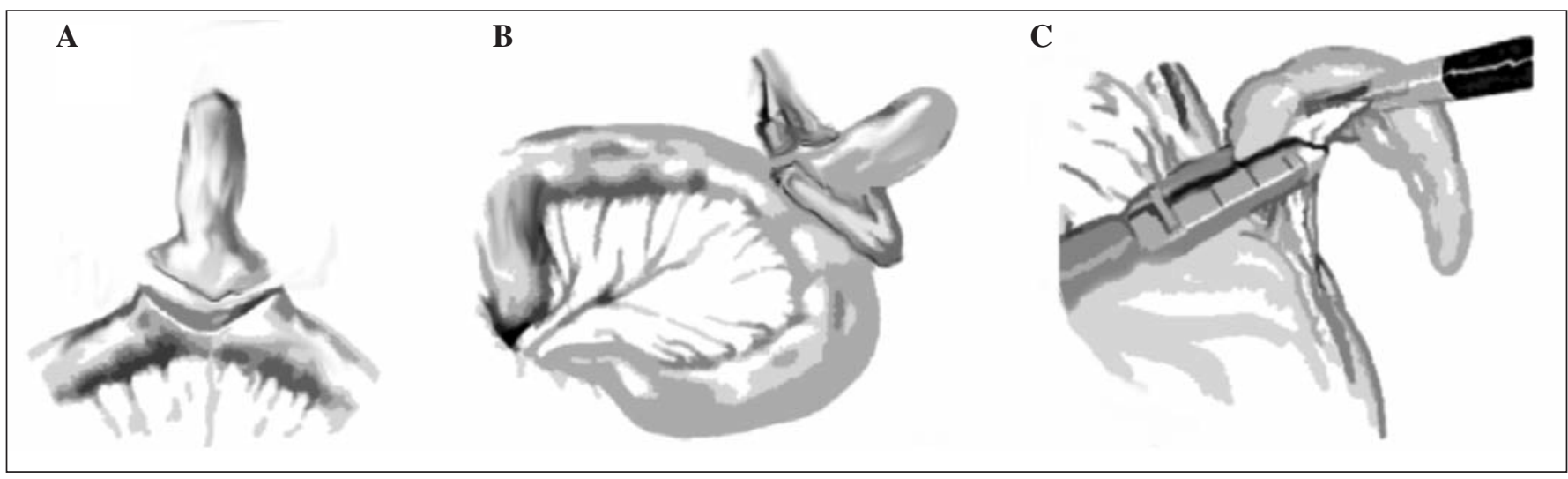

Fig. 5. Techniques employed in diverticulectomy. A. Simple diverticulectomy and enterorrhaphy in laparotomy. B. Diverticulectomy with mechanical stapler after evisceration in the intestinal segment that includes the diverticulum. C. Diverticulectomy via endocutter and endostapler in laparoscopic surgery. 
the inguinal region, $20 \%$ in the femoral, $20 \%$ in the umbilical and $10 \%$ in other locations (10), presenting the typical clinical symptoms, as if it was other portion of the small intestine. The laparoscopic transabdominal (TAPP) and preperitoneal (TEP) approach is currently playing an important role, where the diverticulum is removed and the wall defect repaired comfortably and safely, with an overview of the entire cavity and abdominal wall (10).

Acute inflammation of DM is usually accompanied by fever, vomiting and abdominal pain, and is often indistinguishable from acute appendicitis. Mortality is estimated at around 6\% in elderly patients with delayed diagnosis (2).

When performing additional examinations, the age of the patient and clinical manifestations must be considered. Thus, barium studies are indicated in patients with persistent abdominal pain of unknown origin, positive faecal occult blood without endoscopic findings, or where CT is inconclusive. In paediatric patients with lower gastrointestinal bleeding, the technique of choice is scintigraphy with $\mathrm{Tc}^{99 \mathrm{~m}}$, which provides high sensitivity and specificity in this age group (85 and 95\%, respectively, compared to 63 and $2 \%$ for adults), provided that Meckel's diverticulum is suspected, as the sensitivity of this test generally decreases in lower gastrointestinal bleeding (8). Its usefulness lies in the isotope uptake by ectopic gastric mucosa (as well as the thyroid and salivary gland tissue) (2). The main drawback is that this is not available at all hospitals.

An arteriography may be used in adult patients with episodes of intermittent bleeding or faecal occult blood. Flow in the omphalomesenteric artery is pathognomonic for DM, it usually occurs in an aberrant branch of the superior mesenteric artery (2). The flow must be greater than $0.5 \mathrm{ml}$ per minute to be diagnostic (11). If diagnostic angiography and conventional endoscopy do not reveal the cause of lower gastrointestinal bleeding, capsule endoscopy (14) may be performed, although its value in the acute process is limited (8).

In patients with intestinal obstruction, simple abdominal x-ray may show an enterolith in the lower right quadrant of the image (12). Usually, CT does not accurately show the presence of DM as a cause of obstruction; therefore, presence of DM as the cause of the obstruction is usually confirmed during surgery.

However, for Meckel's diverticulitis without obstruction, CT is the radiological study of choice. The presence of an image with inflammatory changes around a cul-desac, and enteroliths occasionally being appreciated, supports the diagnosis (13). Abdominal ultrasound can be used as a screening technique in patients with acute abdominal pain, as provides a tubular and compressible hyperechoic image (14), which explains the local inflammatory process and indicates emergency surgery. However, it is often misdiagnosed as acute appendicitis.

It is generally agreed that all symptomatic DM cases should be surgically treated, with the laparoscopic approach being the technique of choice (15). It is especially useful in cases where clinical symptoms and findings of additional tests provide results that are indistinguishable from those expected for acute appendicitis (16). At present, there is still controversy about the management of $\mathrm{DM}$ as an incidental finding, which is in general prophylactic diverticulectomy. Although it is not without risk, it is considered justified given the possibility of developing future complications and the presence of intradiverticular tumours, and the possibility of malignisation (17). Based on clinical practice guidelines, resection of DM is recommended in men under 40 years old if it is $>2 \mathrm{~cm}$ in diameter or showing a mesodiverticular band, as these are the ones that are more likely to lead to complications (17).

\section{CONCLUSIONS}

Meckel's diverticulum is much more frequent in children than in adults. The laparoscopic approach is appropriate when DM is suspected, as the entire abdominal cavity can be explored with the minimum possible surgical trauma. We understand that prophylactic appendectomy should be performed in all cases affected by DM. No specialised tests are needed when there is clear clinical suspicion and surgery must be performed without delay in emergency cases. For less urgent cases, abdominal ultrasound and scintigraphy with $\mathrm{Tc}^{99}$ (although it has a high rate of false negatives) are recommended, with the most sensitive and specific being vascular interventional $\mathrm{X}$-ray, which is usually reserved. Given the symptoms of active lower gastrointestinal bleeding or acute abdominal pain, and general malaise and fever, we are forced to rule out the acute diverticular disease and indicate emergency surgery if reasonable suspicion exists.

\section{REFERENCES}

1. Moore TC. Omphalomesenteric duct malformations. Semin Pediatr Surg 1996;5:116-23.

2. Ajaz A. Malik, Shams-ul-Bari, Khrshid A. Wani, Abdul R. Khaja Meckel's Diverticulum-Revisited. The Saudi Journal of Gastroenterology 2010;16(1):3-7.

3. Schwartz MZ. Meckel's diverticulum and other omphalomesenteric duct remnants. In: Wyllie R, Hyams JS, editors. Pediatric gastrointestinal disease: pathophysiology, diagnosis, management. Philadelphia: Saunders; 1999. p. 483-8.

4. Mackey WC, Dineen P. A fifty year experience with Meckel's diverticulum. Surg Gynecol Obstet 1983;156:56-64.

5. Andreyev HJ, Owen RA, Thompson I, Forbes A. Association between Meckel's diverticulum and Crohn's disease: a retrospective review. Gut 1994;35:788-90.

6. Fich A, Talley NJ, Shorter RG, Phillips SF. Does Helicobacter pylori colonize the gastric mucosa of Meckel's diverticulum? Mayo Clin Proc 1990;65:187-91.

7. Groebli Y, Bertin D, Morel P. Meckel's diverticulum in adults: retrospective analysis of 119 cases and historical review. Eur J Surg 2001;167:518-24.

8. Ríos A, Montoya MJ, Rodríguez JM, Parrilla P. Hemorragia digestiva baja severa originada en el intestino delgado. Rev Esp Enferm Dig 2006;98:196-203.

9. Fa-Si-Oen PR, Roumen RM, Croiset van Uchelen FA. Complications and management of Meckel's diverticulum: a review. Eur J Surg 1999;165:674-8. 
10. Biel A, Villalonga R, López de Cenrruzabeitia I, Rodríguez N, Armengol M. Littre s Hernia: unusual find in inguinoescrotal hernial repair. Rev Esp Enferm Dig 2010;102; 506-507

11. Pérez Folqués JE, Fernández Moreno J, Vázquez Ruiz J, Civera Muñoz FJ, Mansilla Molina D, Guirao Manzano J. Meckel s diverticulum as a cause of lower gastrointestinal bleeding in adults. Rev Esp Enferm Dig 2008;100:506-601.

12. Pantongrag-Brown L, Levine MS, Buetow PC, Buck JL, Elsayed AM. Meckel's enteroliths: clinical, radiologic, and pathologic findings. AJR Am J Roentgenol 1996;167:1447-50.

13. Gayer G, Zissin R, Apter S, Shemesh E, Heldenberg E. Acute diverticulitis of the small bowel: CT findings. Abdom Imaging 1999;24: 452-5.
14. Daneman A, Lobo E, Alton DJ, Shuckett B. The value of sonography, CT and air enema for detection of complicated Meckel diverticulum in children with nonspecific clinical presentation. Pediatr Radiol 1998;28:928-32.

15. Rivas H, Cacchione RN, Allen JW. Laparoscopic management of Meckel's diverticulum in adults. Surg Endosc 2003;17:620-2.

16. Rangarajan M, Palanivelu C, Sentihilkhumar R, Madankumar MV Laparoscopic surgery for perforation of Meckel's diverticulum. Singapore Med J 2007;48:e102.

17. Bona D, Schipani LS, Nencioni M, Rubino B, Bonavina L. Laparoscopic resection for incidentally detected Meckel diverticulum. World J Gastroenterol 2008;14:4961-3. 